\title{
Educación y conciencia ambiental en estudiantes de dos colegios técnicos nocturnos de la provincia de Cartago, Costa Rica
}

José Laurian Ramírez Díaz \& Reymond Gutiérrez Arias²

1. Profesor de la Universidad Católica de Costa Rica y del Centro de Capacitación y Desarrollo (CECADES) de la Dirección General de Servicio Civil, Costa Rica.

jramirezd@ucatolica.ac.cr

2. Profesor de la Universidad Católica de Costa Rica y orientador en la Sección Técnica Nocturna del CTP

Llanos de Santa Lucía, Costa Rica.

reymond.gutierrez.arias@mep.go.cr

Recibido: 08 de marzo del 2018

Corregido: 03 de mayo del 2018

Aceptado: 14 de mayo del 2018

\begin{abstract}
Resumen
La educación y conciencia ambiental es un eje transversal de los programas de estudio de educación formal, incluidos los de orientación, que tiene gran relevancia, especialmente por el deterioro que han causado los modelos de consumo actuales en la naturaleza y el equilibrio del ser humano con esta. El presente artículo expone los resultados de un estudio de enfoque mixto sobre la percepción que tienen estudiantes de dos colegios técnicos profesionales- sección nocturna de la provincia de Cartago, Costa Rica, sobre temas como la gestión y compromiso con causas ambientales, la preocupación y conciencia sobre problemáticas ambientales, los programas y campañas conservacionistas, el respeto a la vida y la conciencia sobre el impacto ambiental de las acciones de consumo, con el fin de analizar y proponer criterios de utilidad en ámbitos de educación técnica nocturna, que coadyuven a la inclusión de acciones a favor de la conciencia y el compromiso ambiental dentro de los procesos de formación técnica en poblaciones adultas.
\end{abstract}

Palabras clave: conciencia, educación, ambiente, orientación, liderazgo.

\section{Abstract \\ Education and environmental awareness in students of two technical night schools in Cartago, city Costa Rica.}

Education and environmental awareness it's an important transversal component of the formal study programs, also Vocational Guidance Service at high school, which high relevance because of the spoilage that the contemporary human's consume habits produces in the Earth, and the relation between people and the nature. This article shows the results of mixed investment about the perception of two-night technical high school sections, at Cartago province, in Costa Rica, about themes like compromising and caring of environmental causes, caring and awareness about environmental problems, programs and environmental campaigns, human's respect about all life types, and the awareness about environmental impact of the consume people habits, with the objective to analyze and propose useful criteria, to include actions to promote the compromising and environmental awareness in the adults students at high school technical education.

Key words: awareness, education, environmental, guidance, leadership. 


\section{INTRODUCCIÓN}

Actualmente, la humanidad afronta uno de los desafíos más notables para su supervivencia: la necesidad apremiante de adaptar conductas y hábitos que comulguen con la preservación del ambiente y la naturaleza. Situaciones como la contaminación y tala de bosques, la amenaza a las fuentes de agua potable, la afectación a la atmósfera, entre otras más, plantean una encrucijada que tiene, como elementos críticos, el cambio de modelos conductuales, especialmente consumistas y de explotación de recursos, por otros que comulguen con la sustentabilidad de la vida en el planeta (Avendaño, 2012).

En el ámbito educativo, el Ministerio de Educación Pública de Costa Rica (MEP) ha adoptado políticas estratégicas de educación ambiental, amparado por leyes y reglamentos. Empero, parece que el avance es lento, al menos así visto particularmente, lo que propone una situación por considerar, ya que ciertas actividades relacionadas a la formación técnica profesional derivan en un impacto ambiental, como muchas otras actividades productivas.

Si bien parece que la esperanza de un cambio sustantivo en este aspecto está depositada en la niñez, la realidad actual requiere de un compromiso de todos los grupos etarios. Por ello, la presente investigación planteó como objetivo analizar la percepción de estudiantes de secciones técnicas nocturnas sobre la educación ambiental, en la provincia de Cartago, Costa Rica, para la consideración de insumos que integren el planeamiento y ejecución de acciones educativas afines en los ámbitos educativos respectivos.

Sobre el rol de la educación y la posición del ser humano respecto a las amenazas y repercusiones que poseen sus actividades sobre el ambiente, el humanismo se plantea como un paradigma que, entre otros objetivos, configura la voluntad y el carácter del estudiantado, con base en el entrenamiento de habilidades que les convierte en agentes de cambio, trascendiendo las satisfacciones individuales hacia la ejecución de valores o ideales de nivel superior (Rodríguez, 2013). Se proponen, así, los constructos de conciencia ambiental y conducta sustentable como pilares de la educación ambiental, la cual promueve la construcción de una conciencia planetaria que favorece la capacidad de análisis y reflexión sobre la evolución humana, del planeta y el universo, promoviendo una toma de conciencia del papel del ser humano en el planeta, con base en las repercusiones de sus acciones, propiciando un compromiso con el ambiente, la diversidad y la interculturalidad (Calixto, 2012).

Según Zelezny y Schultz (2000), citados por Tonello y Valladares (2015, p.46), la conciencia ambiental "se refiere a determinados factores psicológicos relacionados con la propensión de las personas a realizar comportamientos proambientales". Gomera (2008) explica que, una parte importante de la conciencia ambiental depende de la toma de decisiones, especialmente de hábitos y conductas armoniosos con la preservación del ambiente, que involucra la dimensión moral y la mejora en la interacción del sujeto con su entorno (Acebal, 2010). En este sentido, Carabais (2002) explica que la construcción y mantenimiento de la conciencia ambiental plantea, frecuentemente, el desafío de encontrar una satisfacción personal más que de reforzamiento social, cuyo ejemplo de esto puede ser el abandono del confort del vehículo por el transporte público, para disminuir el nivel de contaminación particular.

La conducta sustentable es "un conjunto de acciones intencionales y efectivas que resultan en la conservación del ambiente" (Tonello y Valladares, 2015, p.47), que plantea un ámbito de estudio de intervención interdisciplinaria, donde la psicología ambiental se ha interesado por la interacción del individuo con su ambiente (López y García, 2005), la cual analiza percepciones, actitudes y comportamientos de la persona en relación con su contexto físico y social (Moser, 2003).

Respecto al término de sustentabilidad, este se refiere a los estilos de vida que satisfacen las necesidades presentes sin comprometer a las generaciones futuras (Corral y de Queiroz, 2004); Moser (2003) distingue cuatro niveles de intervención: 1) microambiente, considerado en espacios privados como el 
hogar, 2) ambientes de proximidad, tales como el barrio o el lugar de trabajo, 3) ambientes públicos, como las ciudades o pueblos y 4) ambiente global, que considera al planeta.

En cuanto a la disciplina de Orientación Educativa, los programas de estudio de educación formal propuesto por el MEP plantean el concepto de sostenibilidad ambiental desde una perspectiva de educación ciudadana, amparada en la Política Curricular Educar para una nueva ciudananía (acuerdo No 07-64-2016 del Consejo Superior de Educación, sesión No 64-2016 del 17 de noviembre, 2016).

La formación integral de las personas va más allá de la mera transmisión de conocimientos académicos, apuntando más bien, al desarrollo pleno de la persona y sus potencialidades, todo ello sustentado en los pilares: la educación para el desarrollo sostenible, la ciudadanía digital con equidad social y el fortalecimiento de una ciudadanía con identidad nacional. (MEP, 2017, p.11)

Por lo tanto, se infiere que la educación y la conciencia ambiental juegan un papel clave en cada uno de los niveles del sistema educativo: desde educación preescolar hasta el Ciclo de Educación Diversificada (conclusión de la secundaria).

La educación nocturna ronda $11 \%$ del total de la matrícula en secundaria, y plantea situaciones relevantes: 1) la mayor desigualdad en el nivel de secundaria; 2) el aumento de su índice de exclusión intraanual del 18,8\% en 2005 al 21,5\% en 2015, ambos aspectos descritos por el Sexto Informe del Estado de la Educación (Proyecto Estado de la Nación, 2017); 3) el desempeño más bajo en la educación formal costarricense hasta 2014, donde el abandono escolar en las modalidades académica y técnica nocturna rondó $26,9 \%$ y $8,9 \%$ respectivamente; 4 ) la necesidad de trabajo y cuido del hogar que, aunque es una situación compartida por estudiantes de modalidad diurna, se percibe distinto en horario nocturno; 5) la falta de infraestructura propia, que causa que varias instituciones nocturnas la compartan con colegios diurnos, estos últimos puntos tratados en el Quinto Informe del Estado de la Educación (Proyecto Estado de la Nación, 2015). Para finalizar, particularmente se considera que la disminución de la jornada lectiva nocturna con respecto a la diurna, limita la incorporación de ejes transversales como la educación ambiental.

En educación técnica nocturna existe el servicio de Orientación Educativa y Vocacional (OEV), establecido en el Artículo 22 de la Ley Fundamental de Educación (No 2160), de 1957, el cual ha evolucionado durante décadas y donde se destaca, en la actualidad, a la Organización Internacional del Trabajo (OIT) y su concepto de orientación profesional el cual, parafraseando a Hansen (2006), se define como aquellos servicios y actividades que asisten a las personas a lo largo de su vida, para ejecutar opciones educacionales, formativas y laborales, así como en la gestion de las profesiones.

Entre las características y propósitos de la educación técnica se encuentran, por un lado, la posibilidad que ofrece para mejorar la calidad de vida, al plantear una formación que interactúa con el sector productivo $y$, por otro, que propone un escenario para impulsar y fortalecer vínculos entre empresas y el ámbito educativo (Ministerio de Educación Nacional de la República de Colombia, 2016). Por tanto, cada profesional en OEV asume un papel preponderante en la agenda didáctica, ya que plantea un rol holístico, atendiendo las necesidades socioafectivas del estudiantado e integrando distintos elementos del currículo formativo, promocionando así la integralidad.

Entre los elementos por considerar se encuentran, además, la andragogía, entendiendo que la mayoría de estudiantes en esta modalidad son adultos, por lo que sus considerandos plantean una referencia útil para la intervención, que centra su atención en el estudiante como un ser capaz de idear sus propias estrategias de aprendizaje y reconocer sus necesidades y expectativas individuales; es decir, no solo desarrolla conductas, sino aptitudes en un aprendizaje autodirigido, autogestionado y actitudinal (Rodríguez, 2003). 


\section{METODOLOGÍA}

El estudio fue mixto de diseño explicativo secuencial (DEXPLIS). La primera etapa constó de análisis cuantitativo mediante un cuestionario de 84 proposiciones relacionadas con conductas de reciclaje y tratamiento de residuos, así como el cuidado de cualquier forma de vida, entre otras temáticas elegidas a partir de los planteamientos teóricos encontrados en la investigación preliminar, los antecedentes y las leyes y disposiciones del Ministerio de Educación Pública sobre la materia, descritos en el desarrollo del documento.

La cuantificación se dio con base en una escala de Likert de cuatro criterios: siempre, casi siempre, casi nunca y nunca, aplicado a una muestra de 111 estudiantes (véase Tabla 1). La segunda etapa, de corte cualitativo, se basó en dos grupos focales donde se analizaron los resultados estadísticos, con una población de 13 personas (véase Tabla 2).

TABLA 1

Muestreo cuantitativo

\begin{tabular}{cccc} 
& & Frecuencia & Porcentaje \\
\multirow{2}{*}{ Válido } & Hombre & 44 & 39,6 \\
& Mujer & 67 & 60,4 \\
& Total & 111 & 100,0 \\
\hline
\end{tabular}

Fuente: Elaboración propia (2018).

TABLA 2

Muestro cualitativo

\begin{tabular}{|cccc|}
\hline Participante & Género & Edad & Especialidad \\
\hline STC1-1 & Hombre & 30 & Productividad y calidad \\
\hline STC1-2 & Mujer & 30 & Secretariado ejecutivo \\
\hline STC1-3 & Mujer & 27 & Secretariado ejecutivo \\
\hline STC1-4 & Mujer & 29 & Informática empresarial \\
\hline STC1-5 & Hombre & 23 & Productividad y calidad \\
\hline STC1-6 & Hombre & 28 & Informática empresarial \\
\hline STC2-1 & Hombre & 20 & Informática en soporte \\
\hline STC2-2 & Hombre & 19 & Informática en soporte \\
\hline STC2-3 & Hombre & 40 & Informática en soporte \\
\hline STC2-4 & Hombre & 31 & Informática en soporte \\
\hline STC2-5 & Mujer & 22 & Secretariado ejecutivo \\
\hline STC2-6 & Mujer & 19 & Secretariado ejecutivo \\
\hline STC2-7 & Mujer & 22 & Secretariado ejecutivo \\
\hline
\end{tabular}

Fuente: Elaboración propia (2018).

El criterio de selección de participantes fue la condición de estudiante regular de alguna de las secciones técnicas nocturnas escogidas, las cuales presentaron un registro aproximado de 300 discentes en 2017 cada una, sostenido durante los dos años anteriores al estudio, por lo que se les consideró instituciones 
con una matrícula estable en comparación a otras símiles. En los grupos focales participaron estudiantes de forma voluntaria, quienes anteriormente contestaron el cuestionario y cuya información se analizó factorialmente con el software SPSS (véase Tabla 3), obteniendo una varianza total explicada de 67,377\% y, para cada dimensión dada, se verificó su alfa de Cronbach, todos los anteriores como métodos para la validación del mismo. Para el análisis de información cualitativa se utilizó el programa Atlas.ti 8, a partir de la construcción de redes semánticas.

TABLA 3

Prueba de KMO y Bartlett

Medida Kaiser-Meyer-Olkin de adecuación de muestreo

, 816

Prueba de esfericidad de Bartlett

Aprox. Chi-cuadrado

2276,124

gl

703

Sig.

, 000

Fuente: Elaboración propia (2018).

\section{RESULTADOS Y ANÁLISIS}

El análisis factorial derivó en cinco dimensiones o indicadores, con fiabilidad superior a un Alfa de Cronbach $(A C)$ de 0,7 . Se describen a continuación:

\section{Gestión y compromiso con causas ambientales}

Esta dimensión se compuso de 13 ítem referidos a participación en campañas y compromiso de cuidado ambiental y su AC fue de 0,927. Entre los hallazgos parece existir cierta predisposición hacia un compromiso femenino, deducido a partir de la tendencia entre intermedia y elevada que las mujeres presentaron en grado mayor respecto a los hombres (véase Tabla 4).

TABLA 4

Gestión y compromiso ambiental

\begin{tabular}{|c|c|c|c|c|}
\hline \multirow{2}{*}{\multicolumn{2}{|c|}{ Nivel }} & \multicolumn{2}{|c|}{ Género } & \multirow{3}{*}{$\begin{array}{c}\text { Total } \\
6\end{array}$} \\
\hline & & \multirow{2}{*}{$\begin{array}{c}\text { Hombre } \\
5\end{array}$} & \multirow{2}{*}{$\begin{array}{c}\text { Mujer } \\
1\end{array}$} & \\
\hline Bajo & Recuento & & & \\
\hline & $\%$ dentro del indicador & $83,3 \%$ & $16,7 \%$ & $100,0 \%$ \\
\hline \multirow{2}{*}{ Intermedio } & Recuento & 12 & 16 & 28 \\
\hline & $\%$ dentro del indicador & $42,9 \%$ & $57,1 \%$ & $100,0 \%$ \\
\hline \multirow[t]{2}{*}{ Elevado } & Recuento & 22 & 43 & 65 \\
\hline & $\%$ dentro del indicador & $33,8 \%$ & $66,2 \%$ & $100,0 \%$ \\
\hline \multirow[t]{2}{*}{ Total } & Recuento & 39 & 60 & 99 \\
\hline & $\%$ dentro del indicador & $39,4 \%$ & $60,6 \%$ & $100,0 \%$ \\
\hline
\end{tabular}

Fuente: Elaboración propia (2018). 
Para quienes investigaron, el que las mujeres hayan puntuado más en los niveles intermedio y alto que los varones, parece indicar que las primeras conciben la temática con mayor relevancia que los segundos. En el caso de los niveles descritos para el indicador, es probable que una puntuación de nivel elevado indique una mejor comprensión sobre las acciones y su impacto ambiental, así como la responsabilidad con causas referentes a la materia aquí tratada, de modo que los varones podrían mostrar menos involucramiento o compromiso. En los grupos focales se consultó quiénes presentaban mayor compromiso con causas ambientales. Algunas respuestas fueron:

"(...) tal vez las mujeres sí somos más cuidadosas en ciertos aspectos" (STC2-6). / "Yo pienso que las mujeres son más organizadas (...). Los hombres siento que no, (...) son más, eh, dispersos a eso" (STC1-4). / "(...) yo pienso que sí, que la mujer tiene esa vocación" (STC2-3). / "Los hombres actúan por impulso más bien, o sea, como donde alguien empieza algo, ahí lo sigue, pero las mujeres (...) tienen más conciencia" (STC1-4).

Se observó que para los participantes el liderazgo femenino es más validado respecto a la gestión ambiental, entendida como las acciones que presentan, como componente central, el cuidado de la naturaleza, sus recursos y la vida en general, así como el compromiso, derivado en aparentes ideas que las relacionan con capacidades como el cuido del entorno, incluidas las personas. Lo anterior se relaciona con roles sociales asignados culturalmente. Sin embargo, es importante considerar que liderar iniciativas en este sentido debe trascender el género, y definitivamente no puede convertirse en un factor que valide una serie de constructos y estereotipos sociales de roles. Por tanto, llama la atención lo siguiente:

"(...) me parece que es de compromiso, o sea, no importa si yo soy mujer o hombre" (STC2-6). / (...) yo soy muy organizada y me gusta mucho reciclar y el recoger cosas, pero sí he visto también hombres" (STC1-2). / "(...) hombre y mujer se involucran de igual forma" (STC1-5).

Es importante retomar los ámbitos de acción descritos por Moser (2003), donde los ambientes públicos y global cuentan, aparentemente, con mayor participación de hombres pero que, con base en estos hallazgos, parece existir una demanda del liderazgo femenino.

Retomando los postulados andragógicos descritos por Mota (2008), parecería que el autoconcepto, la preparación para el aprendizaje y la orientación para aprender deben considerarse en la educación ambiental. El autoconcepto, según Shavelson et al. (1976), citados por Goñi (2009, p.23), es “la percepción que una persona tiene de sí misma, que se forma a partir de las experiencias y relaciones con el medio, en donde juegan un importante papel tanto los refuerzos ambientales, como los otros significativos", de modo que podría considerarse, desde la OEV, un planteamiento de visión integrada entre hombres y mujeres el compromiso con la educación y la conciencia ambiental, como una tarea igual de relevante para ambos. En el caso de la preparación para el aprendizaje, la cual se "orienta a partir de tareas asociadas a los papeles sociales efectivamente desarrollados por los aprendices" y la orientación para aprender, "que presenta una especial perspectiva en términos de aplicación potencial de los conocimientos en comparación con otra" (Mota, 2008, p.39), podrían ser necesarias como pautas interventivas que estimulen el compromiso entre ambos géneros, ya que si bien parece existir una demanda por el liderazgo femenino, no debe interpretarse como algo exclusivo.

Con base en lo anterior, Castro y Nader (2004, p.47) explican que "los atributos del líder son en su mayoría universales (éticos) y las acciones que lo hacen efectivo trascienden el contexto" (sic), de modo que, tal como puede interpretarse en la información recopilada, por un lado el cuidado de la prole y el rol materno y, por otro, la atribuida atención simultánea de varias situaciones por parte de la mujer, parecieran ser razones para que el liderazgo en la educación ambiental de éstas sea más apreciado, por lo que podrían establecerse estrategias que promuevan el compromiso ético de ambos géneros, validando la 
capacidad de los liderazgos lo cual, a criterio particular, podría plantear una estrategia exitosa, y ser un elemento aliado en las acciones que los profesionales en OEV y el profesorado puedan implementar en este sentido. No se omite decir que la capacidad de influir positivamente en cada uno de los ambientes mencionados por Moser (2003) trasciende al género. Desde el sistema educativo debe decodificarse el esquema de creencias, en caso de encontrar posturas que consideren que la responsabilidad le corresponde a la mujer por los elementos ya analizados, y promover el liderazgo en la educación y conciencia ambiental como una responsabilidad indistinta al género.

\section{Preocupación y conciencia sobre problemáticas ambientales}

Con un 0,895 de AC, la dimensión sumó un total de ocho ítems sobre la preocupación por el calentamiento global, la contaminación de ríos, la tala de bosques y el cambio climático, entre otros.

TABLA 5

Preocupación y conciencia sobre problemáticas ambientales

\begin{tabular}{|c|c|c|c|c|}
\hline \multirow{2}{*}{ Nivel } & & \multicolumn{2}{|c|}{ Género } & \multirow{2}{*}{ Total } \\
\hline & & Hombre & Mujer & \\
\hline \multirow[t]{2}{*}{ Bajo } & Recuento & 0 & 2 & 2 \\
\hline & $\%$ dentro del indicador & $0,0 \%$ & $100,0 \%$ & $100,0 \%$ \\
\hline \multirow[t]{2}{*}{ Intermedio } & Recuento & 4 & 3 & 7 \\
\hline & $\%$ dentro del indicador & $57,1 \%$ & $42,9 \%$ & $100,0 \%$ \\
\hline \multirow[t]{2}{*}{ Elevado } & Recuento & 36 & 58 & 94 \\
\hline & $\%$ dentro del indicador & $38,3 \%$ & $61,7 \%$ & $100,0 \%$ \\
\hline \multirow[t]{2}{*}{ Total } & Recuento & 40 & 63 & 103 \\
\hline & $\%$ dentro del indicador & $38,8 \%$ & $61,2 \%$ & $100,0 \%$ \\
\hline
\end{tabular}

Fuente: Elaboración propia (2018).

Similar a la dimensión anterior, ambos géneros presentaron diferencias en la preocupación y conciencia sobre problemáticas ambientales. En el nivel elevado, las mujeres puntuaron superior a los varones, aunque ellos presentaron mayor puntuación a nivel intermedio, lo que podría indicar que las primeras exponen mayor conciencia sobre las problemáticas que repercuten en el ambiente, y los varones muestran un interés menor, o traducido en acciones concretas mas no continuas. Lo anterior podría explicar que, según este indicador, es probable que exista un compromiso mayor entre las mujeres, aspecto que debe ser fortalecido en los varones. Al respecto, en los grupos focales se rescataron elementos sobre roles culturales y tradicionales relacionados a la femineidad. Al preguntarles si ellas se preocupaban más, respecto a los hombres, por problemáticas como el cambio climático, la contaminación o tala de bosques, indicaron:

“(...) la mujer trae una naturaleza de madre, de cuidar, de proteger, ella se preocupa más por cuidar y proteger, porque ella sabe lo que es cuidar" (STC1-6). / "(...) las mujeres, tratan de, diay, de ser mejor, de llevar las cosas más ordenadas, ser mejores, y sí, como dicen las compañeras: los hombres siempre, diay, si lo impulsan ellos van, sino no arrancan (...)" (STC1-5). / "(...) las mujeres somos muy organizadas y siempre estamos buscando la manera de, de poder reciclar (...)" (STC1-3). /"(...) es de crianza, o sea, es como que uno ya trae ese chip tiene que hacer todo uno, hacer lo que puede" (STC2-6). 
Parece ser que, similar a la primera dimensión, se tiende a acreditar a la mujer una mayor preocupación por problemáticas ambientales por roles sociales, la maternidad y el cuidado de su entorno. Esta situación parece evidenciarse en distintos ámbitos, lo cual llama la atención y, según el criterio particular, requiere de cuidado al diseñar programas y políticas de educación ambiental. Con respecto a las apreciaciones citadas, Téllez (2005) expone que, durante un estudio realizado en Perú, se evidenció que las mujeres tienden a participar más en actividades relacionadas con el ambiente, aún a pesar de sus múltiples tareas, aunque los hombres también se incorporan. En España se han implementado, también, políticas de educación ambiental que buscan sacar provecho del género, tomando en cuenta el sentir de las mujeres por los derechos de las personas, las relaciones de calidad, las alianzas entre ellas y los varones, así como la reconstrucción del equilibrio con el entorno (Limón y Solís, 2013). En Costa Rica, en las culturas aborígenes, la mujer es quien hereda los derechos y responsabilidades sobre los bienes ya que, se cree, ella es mejor administradora (Almengor, 2017).

No fue un criterio de interés en el estudio analizar los hallazgos desde una perspectiva de género, sin embargo, y al tratarse de un hecho concurrente, se consideró necesario rescatar la importancia que se le asigna a la mujer y valorar los aportes y ventajas que puedan derivar de su liderazgo. Es posible que, al plantear estrategias de educación y conciencia ambiental, su protagonismo sea un elemento importante de retomar ya que, socialmente, parece tener ventajas de tipo moral y cultural, aunque la responsabilidad atañe a ambos géneros.

\section{Programas y campañas conservacionistas}

El tercer indicador obtuvo un AC de 0,719 y constó de ítems referidos a la importancia de causas conservacionistas y campañas ambientales. La información obtenida reveló que, al igual que con los indicadores anteriores, las mujeres tienden a puntuar más alto que los varones, aunque en este caso la diferencia presentó niveles cuantitativamente menores (véase la Tabla 6).

TABLA 6

Programas y campañas conservacionistas

\begin{tabular}{llccc}
\multirow{2}{*}{ Nivel } & & \multicolumn{2}{c}{ Género } & Total \\
\cline { 3 - 4 } Intermedio & Recuento & Hombre & Mujer & \\
& \% dentro del indicador & 4 & 0 & 4 \\
\multirow{2}{*}{ Elevado } & Recuento & $100,0 \%$ & $0,0 \%$ & $100,0 \%$ \\
\cline { 2 - 4 } & \% dentro del indicador & 35 & 65 & 100 \\
\hline \multirow{2}{*}{ Total } & Recuento & $35,0 \%$ & $65,0 \%$ & $100,0 \%$ \\
& \% dentro del indicador & 39 & 65 & 104 \\
\hline
\end{tabular}

Fuente: Elaboración propia (2018).

Las mujeres mantienen la tendencia hacia un nivel elevado respecto a los varones en este indicador. Esto podría explicarse, relacionado con los indicadores anteriores, que ellas participan más activamente respecto a ellos en actividades que tengan como objetivo la gestión ambiental. Para los autores del estudio, esto puede traducirse en que la mujer es más dinámica en actividades colectivas de este tipo que los varones, quienes podrían mostrar una conducta más individualizada o, en su defecto, una postura más pasiva ante la mayor predisposición que parece distinguirse en las féminas respecto a los hombres 
en los temas analizados. En los grupos focales se conversó sobre la relevancia de estas campañas, así como sobre la necesidad del fortalecimiento del proceso de educación ambiental en el país:

“(...) estas campañas son muy importantes para la clasificación de residuos, lo que pasa es que nos hace falta un poquito más en el aspecto (...)" (STC1-4). / "(...) evita esta clase de problemática que hemos tenido (...) tirar las cosas a los ríos, de tirar las cosas al mar, de tirar las cosas a la calle" (sic) (STC1-5). / "(...) empieza desde poquito, desde que yo siento y pienso que estoy las haciendo las cosas bien" (sic) (STC2-6).

Asimismo, se recalcó la importancia que tiene el inicio de la educación ambiental desde los primeros estadios de la infancia:

"(...) sería muy chiva que en las escuelas y el kínder, en esos grados también se centren en eso" (STC2-6) / "(...) debería de la casa primero, y después de las instituciones públicas, deberían de hacer las campañas de reciclaje también" (STC1-4).

Lo anterior es importante ya que, por un lado, ambas son manifestaciones de mujeres, lo cual parece coincidir con la línea hasta ahora analizada sobre su liderazgo, preocupación y compromiso con la educación y la conciencia ambiental y, por otro, con la necesidad que existe en el país de una adecuada gestión en este sentido, la cual está considerada por el MEP desde el Programa Integrado de Educación para el Desarrollo Sostenible y la Gestión Ambiental Institucional, el cual apunta hacia actividades, productos y contenidos orientados a alentar la responsabilidad de las comunidades educativas con el desarrollo sostenible, así como la promoción de una cultura responsable y consciente con el ambiente (Alfaro, 2015). A la vez existe, como eje transversal, la política Cultura Ambiental para el Desarrollo Sostenible pero, tal y como explica Mata (2013, p.59), "es débil, ya que al ser un eje transversal que no tiene contenidos curriculares, su enseñanza depende del grado de conciencia ambiental de cada maestro y de la disposición que tenga cada uno para transmitir sus conocimientos a los estudiantes", por tanto es necesario educar a las familias, a los estudiantes y a las comunidades.

Con respecto a la gestión y al cuidado ambiental en las comunidades, sean educativas o de vecinos, es importante considerar alianzas con organizaciones que, además de gestionar los residuos adecuadamente, establezcan políticas de educación y, como condición relevante propuesta por los participantes, que incentiven dichas prácticas. Así, las municipalidades pueden jugar un papel de suma relevancia y han demostrado ya su eficacia en distintos países (Galarza, 2012). En Costa Rica, en 2014, se generaron cuatro mil toneladas diarias de residuos sólidos ordinarios; con la Ley de Gestión Integral de Residuos Sólidos (No 8839), para el 2015, unos 64 cantones (72\% del total) contaban con el Plan Municipal de Gestión Integral de Residuos Sólidos (GIRS) y 47 (54\%) con oficinas de gestión ambiental (Central America Data, 2015). A criterio particular, estos avances son relevantes, pero deben tener mayor promoción. Para quienes participaron en los grupos focales, no existe información suficiente sobre el reciclaje:

"Hay mucha gente que dice: 'yo estoy reciclando', pero es que todo lo está poniendo junto, entonces diay, tampoco es la ciencia, tenemos que saber clasificar los residuos también" (STC1-4). / "Hay gente que no sabe qué es tetrabrik" (STC2-2).

Es importante no sólo la realización de campañas de reciclaje y cuidado del ambiente, sino el socializar su gestión a nivel comunal, de modo que las personas conozcan sus alcances y, más importante, sean sujetos de aprendizaje y formación colectiva, de modo que puedan participar y sacar el máximo provecho. Es fundamental recalcar que, aludiendo a los programas de estudio del servicio de OEV, una de las recomendaciones dadas por un estudiante fue que se incorporaran a la evaluación este tipo de acciones: 
"(...) que sea parte de la nota. Que sea evaluada, que tenga un porcentaje" (STC2-3). Se hizo la consulta de cuáles departamentos o instancias deberían involucrase en estas políticas, los profesionales en OEV fueron considerados idóneos para ello por uno de los participantes del grupo focal, al decir: "Orientación. Y que den las herramientas, digamos, que expliquen bien, que al alumno le quede claro qué es reciclar, cómo hacerlo, en dónde... y que haya los lugares adecuados en el colegio para hacerlo" (STC2-3).

\section{Respeto a la vida}

Esta dimensión se derivó del respeto a la vida en general, incluyendo la no humana. Con un total de tres ítems, obtuvo un AC de 0,730 (véase Tabla 7).

TABLA 7

Respeto a la vida

\begin{tabular}{llccc}
\multirow{2}{*}{ Nivel } & & \multicolumn{2}{c}{ Género } & Total \\
\cline { 3 - 4 } Bajo & Recuento & Hombre & Mujer & \\
& \% dentro del indicador & 2 & 0 & 2 \\
\multirow{2}{*}{ Elevado } & Recuento & $100,0 \%$ & $0,0 \%$ & $100,0 \%$ \\
\cline { 2 - 4 } & \% dentro del indicador & 40 & 65 & 105 \\
\hline \multirow{2}{*}{ Total } & Recuento & $38,1 \%$ & $61,9 \%$ & $100,0 \%$ \\
& \% dentro del indicador & 42 & 65 & 107 \\
\hline
\end{tabular}

Fuente: Elaboración propia (2018).

Similar a los indicadores anteriores, las mujeres puntúan más a nivel elevado que los varones $(61,9 \%$ y $38,1 \%$ respectivamente), lo que podría indicar que, además de las gestión y compromiso ambiental, la preocupación por problemas y la filiación con campañas de preservación del medio y el valor de la vida es mayor en ellas, lo que podría ser una situación que explique por qué parece darse un interés mayor en las consideraciones tratadas en ellas respecto a los hombres: el reparo transversal de la vida como condición más amenazada por el deterioro ambiental. Aunque pareció existir una elevada tendencia hacia la consideración de los animales dentro del indicador, en los grupos focales se explicó la preeminencia que tienen todas las formas de vida:

"Yo siento que aparte que el respeto es parte de nosotros, si no hubieran árboles no podríamos tener el oxígeno, si no hubieran plantas, si no estuvieran los animales, verdad, entonces es parte de nuestra vida también, que hay que respetar" (Sic) (STC1-2). / "Es más que todo respeto a la vida en general" (STC2-2). / "Es importantísimo, porque yo pienso que una persona que no respeta a los animales no se respeta él primero (...)" (STC2-3). / "Yo tengo que respetar a la vida como, o sea, todo lo que me rodea, no solo a los seres humanos, todos los seres, todo, el agua, todo todo" (STC2-6).

Es poco lo que debe analizarse al respecto según la percepción particular, no por falta de información o referentes teóricos, sino porque el respeto hacia la vida en general es un componente de gran relevancia. Sobre la educación ambiental Rengifo, Quiquiatez y Mora $(2012$, p.4) explican que "es un proceso que reconoce valores y aclara conceptos centrados en fomentar las actitudes, destrezas, habilidades y aptitudes necesarias para comprender y apreciar las interrelaciones entre el ser humano, su cultura y la interrelación con la naturaleza". El respecto por la vida en general es un elemento de suma relevancia e inherente a toda acción promotora de la educación y la conciencia ambiental. 


\section{Conciencia sobre el impacto ambiental}

La última dimensión analizada, con un AC de 0,749 y compuesta de dos ítems, estuvo relacionada con el conocimiento de las personas sobre el impacto de sus acciones en el ambiente.

TABLA 8

Conciencia sobre el impacto ambiental individual

\begin{tabular}{llccc}
\multirow{2}{*}{ Nivel } & & \multicolumn{2}{c}{ Género } & Total \\
\cline { 3 - 4 } Bajo & Recuento & Hombre & Mujer & \\
\cline { 3 - 4 } & \% dentro del indicador & 4 & 4 & 8 \\
\hline \multirow{2}{*}{ Intermedio } & Recuento & $50,0 \%$ & $50,0 \%$ & $100,0 \%$ \\
\cline { 2 - 4 } & \% dentro del indicador & 12 & 20 & 32 \\
\hline Total & Recuento & $37,5 \%$ & $62,5 \%$ & $100,0 \%$ \\
& \% dentro del indicador & 18 & 22 & 40 \\
\hline
\end{tabular}

Fuente: Elaboración propia (2018).

Respecto a este indicador, se observó que los niveles por género tienen un comportamiento similar a los anteriores: las mujeres plantean un mayor conocimiento del impacto que causan sus hábitos en el ambiente respecto a los hombres, aunque llama la atención que, el nivel predominante en ellas es el intermedio, lo cual podría estar relacionado con el indicador tras anterior, en tanto consideran que la información sobre el cuidado ambiental es escasa. Es posible que esta "desinformación" también se dé en este caso. Sin embargo, parece que el impacto de ciertas campañas sociales, sean formales o no, favorecen la creación de conciencia ambiental. Algunas manifestaciones al respecto fueron:

“Diay, básicamente empezando por las bolsas plásticas, por pedir una pajilla que es malísima también (...) todo lo que uno en realidad consume de más que no debería tener" (STC1-2). / "(..) si yo voy ahí al super a comprar lo que sea, me llevo mi bolso o no pido bolsa, si me lo puedo traer en la mano, lo traigo en la mano" (STC1-1). / "(...) si lo que me lo puedo traer en la mano me lo traigo y si no trato de ver que todo entre en una sola bolsa (...)" (STC1-4). / "(...) ahorrar agua me costó muchísimo porque, diay, uno ya tiene ese chip" (STC2-6). "Diay tiene un impacto grande por lo mismo, por todos los desperdicios, por el avance de la tecnología" (STC1-1).

Es importante señalar que ciertas cadenas de restaurantes y supermercados han puesto en marcha campañas para la erradicación del uso de la pajilla y de las bolsas plásticas, lo cual podría estar incidiendo positivamente. Empero, estas acciones deben prevalecer en el tiempo para mejorar su impacto y, de ser posible, crear una cultura de réplica y estímulos a nivel social. Dentro del marco jurídico costarricense, en respuesta a la Ley 8839 para la Gestión Integral de Residuos, el MEP (2011) dispuso del Programa Nacional para la Gestión Integral de Residuos Sólidos, el cual dispone, entre otras acciones, del diagnóstico institucional del tratamiento de los residuos sólidos, la disposición de lugares para el depósito de los mismos según su clase y la sensibilización a la comunidad educativa. Vale aquí recalcar lo expuesto por Mata (2013), para quien la educación ambiental queda sujeta a la voluntad del personal que labora en el centro educativo, de modo que debe incentivarse, como una prioridad, su compromiso activo al respecto. 


\section{CONCLUSIONES}

Parece que, para una adecuada educación ambiental, deben valorarse acciones que inicien por el establecimiento de liderazgos claros dentro de las instituciones, donde no se asignen a las personas por puestos o roles, con base en sus capacidades o experiencia solamente, sino que sean formadas, entrenadas y motivadas en el ejercicio de sus habilidades y la mejora de la gestión e impacto de sus acciones, además de involucrar a la comunidad en ellas, estableciendo incentivos tanto internos como externos.

Es importante considerar la aparente ventaja que tiene el liderazgo femenino, relacionado a ciertos roles asignados a la mujer, pues socialmente goza de mayor credibilidad y validez para dirigir y liderar acciones de educación y conciencia ambiental. Esto no debe verse como una situación que amenace a la equidad de género, si es que así fuera interpretado por ciertas organizaciones o sectores, sino que podría concebirse como una oportunidad para alcanzar objetivos urgentes, en consideración a la realidad actual. Es evidente, y particularmente oportuno, que los hombres también asuman liderazgo en tan importante problemática, pero que para esto se apueste por nuevas estrategias que planteen una eficacia mayor en el logro de los objetivos.

La educación ambiental nunca había sido tan importante como ahora, y es necesario aplicarla en todos los ámbitos posibles. Delegar las acciones a terceros no ha sido una estrategia que haya funcionado en el pasado o el presente, por tanto es descartable para el futuro. Todas las personas tienen responsabilidad en la implementación de soluciones, especialmente aquellas que, por vocación, forman a otras. Promover liderazgos eficaces parece ser una estrategia para alcanzar los objetivos de la educación ambiental, como eje transversal en contextos de educación técnica nocturna.

\section{REFERENCIAS}

Acebal, M. (2010). Conciencia ambiental y formación de maestras y maestros. Universidad de Málaga, Didáctica de la Matemática, Ciencias Sociales y Ciencias Experimentales. Málaga: SPICUM servicio de publicaciones.

Alfaro, A. (1 de junio, 2015). Sostenibilidad ambiental: pilar de la nueva política educativa. Recuerado de http://www.mep.go.cr/noticias: http://www.mep.go.cr/noticias/sostenibilidad -ambiental-pilar-nueva-politica-educativa

Almengor, K. (5 de noviembre, 2017). Entrevista personal. (Ramírez, J., Entrevistador)

Avendaño, W. (2012). La educación ambiental (EA) como herramienta de la responsabilidad social (RS). Luna Azul, (35), 94-115.

Calixto, R. (2012). Investigación en Educación Ambiental. Revista Mexicana de Investigación Educativa, 17(55), 1019-1033.

Carabais, V. (2002). Conciencia ambiental y comportamiento ecológico: Un análisis de la escala GEB (General Ecological Behavior) de Kaiser. Revista Internacional de Sociología, 1-26.

Castro, A. \& Nader, M. (2004). Estilos de liderazgo, contexto y cultura organizacional. Un estudio comparativo en población civil y militar. Boletín de psicología, (82), 45-63.

Central America Data. (13 de marzo, 2015). Basura en Costa Rica: Otra comisión. Recuperado de https://www.centralamericadata.com/: https://www.centralamericadata.com/es/article/home/ Basura_en_Costa_Rica_Otra_comisin

Corral, V. \& De Queiroz, J. (2004). Aproximaciones al estudio de la conducta sustentable. Medio Ambiente y Comportamiento Humano, $5(1$ y 2), 1-26. 
Galarza, G. (2 de abril, 2012). Un 2\% másde residuos esreciclado debido aincentivo del MEF. El Comercio, p.A12.

Gomera, A. (2008). La conciencia ambiental como herramienta para la educación ambiental: conclusiones y reflexiones de un estudio en el ámbito universitario. (Trabajo Final de Graduación para obtener el Doctorado en Innovación Curricular y Práctica Psicosocioeducativa, Universidad de Córdoba). Córdoba, España. Recuperado de http://www.mapama.gob.es: http://www.mapama.gob.es/es/ ceneam/articulos-de-opinion/2008_11gomera1_tcm7-141797.pdf

Goñi, E. (2009). El autoconcepto personal: estructura interna, medida y variabilidad. España: Servicio Editorial de la Universidad del País Vasco.

Hansen, E. (2006). Orientación Profesional. Un manual de recursos para países de bajos y medianos ingresos. Montevideo: CINTERFOR.

Limón, D. \& Solís, C. (2013). Educación Ambiental y enfoque de género, claves para su integración. Investigación en la escuela, (83), 37-50.

López, B. \& García, E. (2005). Calidad de vida y medio ambiente. La psicología ambiental. Universidades, (30), 9-16.

Mata, A. (25 de marzo, 2013). ONU Medio Ambiente. Recuperado de http://www.pnuma.org/educamb/ reunion_foro_internacional/Informe_Final-Educacion_Ambientalen_Costa_Rica_JICA\%283\%29. pdf

Ministerio de Educación Nacional de la República de Colombia. (2016). Educación técnica y tecnológica para la competitividad. Bogotá, Colombia.

Ministerio de Educación Pública. (2011). Sitio Web del Ministerio de Educación Pública. Recuperado de http://www.mep.go.cr/: http://www.mep.go.cr/sites/default/files/page/adjuntos/programa-nacional-manejo-residuos-mep.pdf

Ministerio de Educación Pública. (2017). Programas de Estudio de Orientación. Primero, Segundo y Tercer Ciclos de la Educación General Básica y Educación Diversificada. [Versión digital]. San José, Costa Rica.

Moser, G. (2003). La Psicología Ambiental en el Siglo 21: El Desafío del Desarrollo Sustentable. Revista de Psicología de la Universidad de Chile, 12(2), 11-17.

Mota, R. (2008). Inclusión Social, Andragogía y Universidad Abierta del Brasil. Educación Superior y Sociedad, 13(1), 32-47.

Proyecto Estado de la Nación. (2015). Quinto Informe del Estado de la Educación. San José: Servicios Gráficos, A.C.

Proyecto Estado de la Nación. (2017). Sexto Informe del Estado de la Educación. San José: Servicios Gráficos, A.C.

Rengifo, B., Quiquiatez, L. \& Mora, F. (2012). La educación ambiental, una estrategia pedagógica que contribuye a la solución de la problemática ambiental en Colombia. Recuperado de http://www.ub.edu/ web/ub/ca/: http://www.ub.edu/geocrit/coloquio2012/actas/06-B-Rengifo.pdf

Rodríguez, J. (2013). Una mirada a la pedagogía tradicional y humanista. Presencia universitaria, 3(5), 36-45.

Rodríguez, P. (2003). La andragogía y el constructivismo en la sociedad del conocimiento. Laurus, 9(15), 80-89.

Téllez, E. (2005). Ministerio de Agricultura y Pesca, Alimentación y Medio Ambiente de España. Recuperado de http://www.mapama.gob.es/: http://www.mapama.gob.es/es/ceneam/articulos-de-opinion/2005_03trellez_tcm7-53026.pdf

Tonello, G. \& Valladares, N. (2015). Conciencia ambiental y conducta sustentable relacionada con el uso de energía para iluminación. Gestión y ambiente, 18(1), 45-59. 